EPJ Web of Conferences 66, 02096 (2014)

DOI: $10.1051 /$ epjconf/ 20146602096

(C) Owned by the authors, published by EDP Sciences, 2014

\title{
Nuclear structure and reaction studies with exotic nuclei at the FRS-ESR
}

\author{
Christoph Scheidenberger ${ }^{1,2 a}$ \\ ${ }^{1}$ GSI Helmholtzzentrum für Schwerionenforschung GmbH, 64291 Darmstadt, Germany \\ ${ }^{2}$ II. Physikalisches Institut, Justus-Liebig-Universität Gießen, 35392 Gießen, Germany
}

\begin{abstract}
Nuclear physics with exotic nuclei in storage rings was pioneered at the SISESR facility in combination with the fragment separator FRS. Already the first experiments in the early 1990s gave access to ground-state properties like masses and half-lives and indicated the research potential of this novel instrumental approach. Many new data have been obtained and interesting phenomena have been explored, e.g. the mass surface was mapped over large areas of the chart of nuclei, isomer studies of longlived states (with half-lives of the order minutes) became possible, the modification of decay properties for highly-charged, high-Z exotic nuclei was observed, and new decay modes, like the beta-decay to bound final states, were studied for the first time. A few years ago, direct reaction experiments on internal targets using inverse kinematics were started: transfer and pickup reactions of astrophysical interest were performed with stable isotopes at energies approaching the Gamow-window, while elastic and inelastic scattering experiments were performed with secondary beams of the unstable doubly magic isotope ${ }^{56} \mathrm{Ni}$ quite recently. In this contribution, the achievements will be reviewed and recent results will be presented. It is dedicated to Paul Kienle.
\end{abstract}

\section{Introduction}

In the second half of the last century, the advent of particle accelerators has prompted the development of storage and cooler rings. These are highly specialized instruments for particle, hadron and nuclear physics as well as atomic physics and various applications. In the 1980s, the production and separation of exotic nuclei by in-flight separation was pioneered at intermediate energies $(30 \ldots 80 \mathrm{MeV} / \mathrm{u})$ at GANIL [1]. This concept was adapted to relativistic energies when the FRS at GSI was proposed by G. Münzenberg and H. Geissel [2], and it was the ingenious spirit of Paul Kienle, at that time scientific director of GSI and responsible for the SIS-ESR project [3], who immediately recognized the tremendous emerging research potential when the fragment separator FRS was combined with the experimental storage ring ESR. This was the origin of an unprecedented nuclear-physics programme, that allows the study of ground-state properties, isomeric states and decay phenomena of highly-charged exotic nuclei [4]. In addition the scattering from internal targets and nuclear reaction experiments with exotic nuclei were on the horizon from the very beginning. Still

${ }^{\text {a }}$ Corresponding author: c.scheidenberger@gsi.de

This is an Open Access article distributed under the terms of the Creative Commons Attribution License 2.0, which permits unrestricted use, distribution, and reproduction in any medium, provided the original work is properly cited. 
today, some 25 years later, the combination of FRS and ESR is unique and yields exciting new developments and results, some of which are featured in the present contribution.

\section{Production, separation, storage and cooling}

At the FRS-ESR exotic nuclei are produced in nuclear reactions (fragmentation, fission) from intense (recently more than $10^{10}$ uranium-ions per second were extracted from the synchrotron driver accelerator) high-energy (typical energies range from $\sim 100 \mathrm{MeV} / \mathrm{u}$ up to $\sim 1 \mathrm{GeV} / \mathrm{u}$ ) stable-ion beams (from hydrogen up to uranium) in a production target at the entrance of the fragment separator FRS [5]. The exotic nuclei produced are separated in-flight, and depending on the ion-optical mode of the FRS, mono-isotopic beams or cocktail beams can be injected into the experimental storage ring ESR [6], depending on the goal of the measurement. After injection, the exotic nuclear beams can be cooled by stochastic pre-cooling (which reduces the momentum spread within several hundred milliseconds to $\delta \mathrm{p} / \mathrm{p} \sim 10^{-3}$ ) and subsequently by electron cooling [7]. When short cooling times ( $\sim 1$ second) are involved, stochastic pre-cooling is essential for "hot" fragment beams, since the electron cooling time increases proportional to $\Delta \mathrm{v}^{3}$, where $\Delta \mathrm{v}$ denotes the velocity difference of the ions and cooler electrons. After electron cooling, all stored ions circulate with the same velocity $\left(\delta \mathrm{v} / \mathrm{v} \sim 10^{-7}\right)$ due to the repeated interaction with the ,cold“ cooler electrons, which they traverse with a circulation frequency of about $1 \ldots 2 \mathrm{MHz}$. Production, storage and cooling are illustrated in figure 1 .

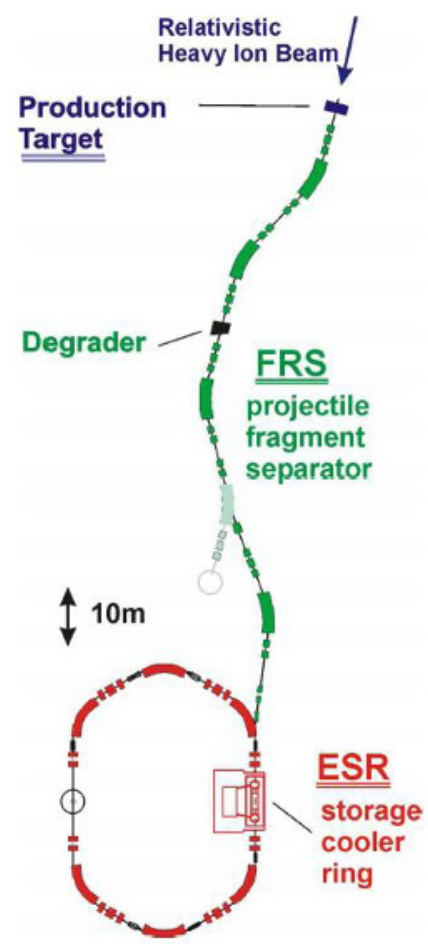

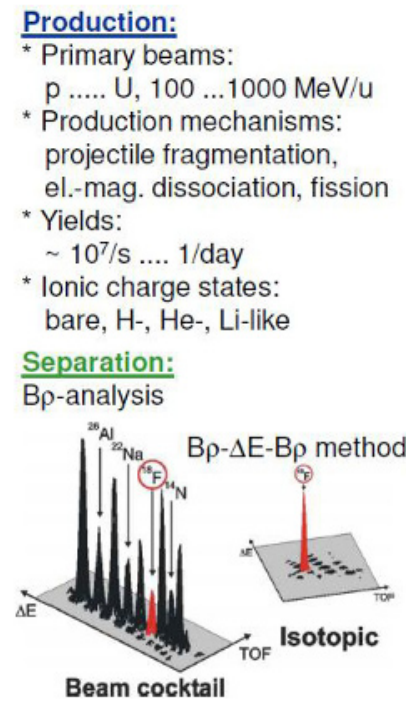

Storage+cooling:

${ }^{*}$ Fast injection (bunch 400ns)

* Storage times: min. .... days

* Cooling: stochastic (pre-)cooling, electron cooling
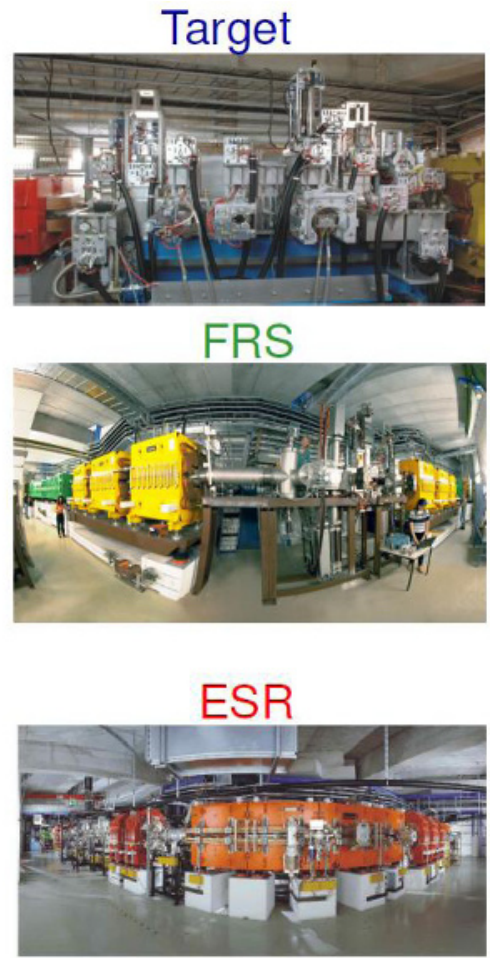

Figure 1. Left: topographic view of fragment separator FRS, including the production-target area at its entrance, and experimental storage and cooler ring ESR of GSI. Center: production and properties of exotic nuclei, beam separation modes available at the FRS, and storage and cooling characteristics. Right: photographs of the target area, the separator central focal plane, and one bending section of the storage ring. 


\section{The pioneering experiments}

The pioneering experiments with unstable beams were carried out in the early 1990s and provided a number of remarkable results. The first stored and cooled relativistic fragment beams were ${ }^{18} \mathrm{~F}$ and ${ }^{19} \mathrm{Ne}[8]$ and demonstrated the feasibility of this new experimental concept. In the same year, the betadecay to bound final electronic states, already predicted in 1947 by Daudel et al. [9], was discovered with fully stripped beams of ${ }^{163}$ Dy [10]. In a next step, half-life measurements on bare, mass-resolved isomers were performed, which showed changes in the half-life depending on the ionic charge state [11]. With these experiments, the foundation of Schottky Mass Spectrometry was laid. The coasting ions induce a Schottky-noise signal when passing the pair of capacitive pickup plates mounted inside the storage-ring aperture. After Fourier-transformation, each stored ion species produces signals ('Schottky lines') at distinct frequencies corresponding to the revolution frequencies of the ion species (including higher harmonics), determined by their mass-to-charge ratio. The data are recorded and processed using a spectrum analyzer or off-line analysis. This technique, later refined to time-resolved Schottky Mass Spectrometry, was the work horse of the following decade. With stored and cooled cocktail beams, large areas of the nuclear mass surface can be measured with high accuracy in a few experiments [12]. The limiting parameter, which restricts the accessible half-lives of nuclei under study to approximately one second, is the cooling time needed to reduce the longitudinal phase space of the "hot" projectile fragments. This limitation can be circumvented with Isochronous Mass Spectrometry [13], which gives access to nuclei and isomeric states with half-lives of the order of micro-seconds [14]. This method employs the isochronous ion-optical mode of the ESR [15] together with multiple time-of-flight measurements of the ions, where the velocity differences of one particular ion species are compensated by different path lengths such that they have equal revolution times. Finally, the bound-state beta decay in radioactive nuclei was studied, where particular interest was devoted to the measurement of the branching ratio of bound and continuum beta decay [16]. Such measurements profit from a suitable choice of isotopes, so that lifetimes and decay Q-values fit the experimental conditions and goals.

\section{Recent results}

\subsection{Mass and half-life measurements}

When the ESR is used as a multi-turn mass spectrometer, the masses of exotic nuclei from the FRS can be determined to high accuracy. With the two complementary methods, Schottky-MassSpectrometry (SMS) [17] and Isochronous-Mass-Spectrometry (IMS) [13] the masses of almost 400 isotopes were measured accurately for the first time, some 300 mass values were improved, and overall about 1100 different nuclides were observed, which is approximately $40 \%$ of all known isotopes. Typically a mass precision of $10 \ldots 100 \mathrm{keV} / \mathrm{c}^{2}$ is obtained, so that the data provide important input for exploratory nuclear structure studies, astrophysical applications and for improving nuclear theories and mass models. Due to the continuously increasing intensity of uranium beams, neutronrich nuclei can be reached efficiently, and even new isotopes can be identified by an accurate mass-tocharge ratio measurement, which allows for unambiguous identification [18]. With the new data, the average interaction strength of protons and neutrons was investigated for different isotopes around the double magic nucleus ${ }^{208} \mathrm{~Pb}$. It could be shown, that the interaction is determined by the spatial overlap of proton and neutron wave functions, so that the interaction is strong for particle-particle and hole-hole states and weak for particle-hole and hole-particle configurations [18].

Lifetime experiments were performed with time-resolved Schottky-Mass-Spectrometry and with particle detectors located behind the dipole sections downstream of the straight sections of the ESR. As ions can be stored over long periods of time (light ions can be stored for many hours or even days) decay properties can be studied, and as the ions are produced at relativistic speed, their electrons are stripped off and they prevail as bare, H-like or He-like ions. This has consequences for the beta-decay half-lives of ground and isomeric states, and there are several examples where decay "constants" are 
altered drastically, and decay times are increased or decreased depending on the electronic configuration of the highly-charged ions [19]. Recent interest was attracted when an oscillatory modulation of the exponential decay curves was claimed [20]. While there are several possible explanations, their origin needs to be clarified in forthcoming experiments.

The capability of time-resolved Schottky spectroscopy, which intrinsically combines non-destructive observation and continuous intensity measurements with high mass resolving power, opens up the novel opportunity to study long-lived isomers, whose lifetime is too long to be measured with conventional prompt gamma-ray spectroscopy and where ground and excited states cannot be separated by conventional separation or identification methods and/or where the produced quantities are too low for a conventional decay-curve measurement. This has led to the discovery of new isomeric states and new decay branches [21], see figure 2 .

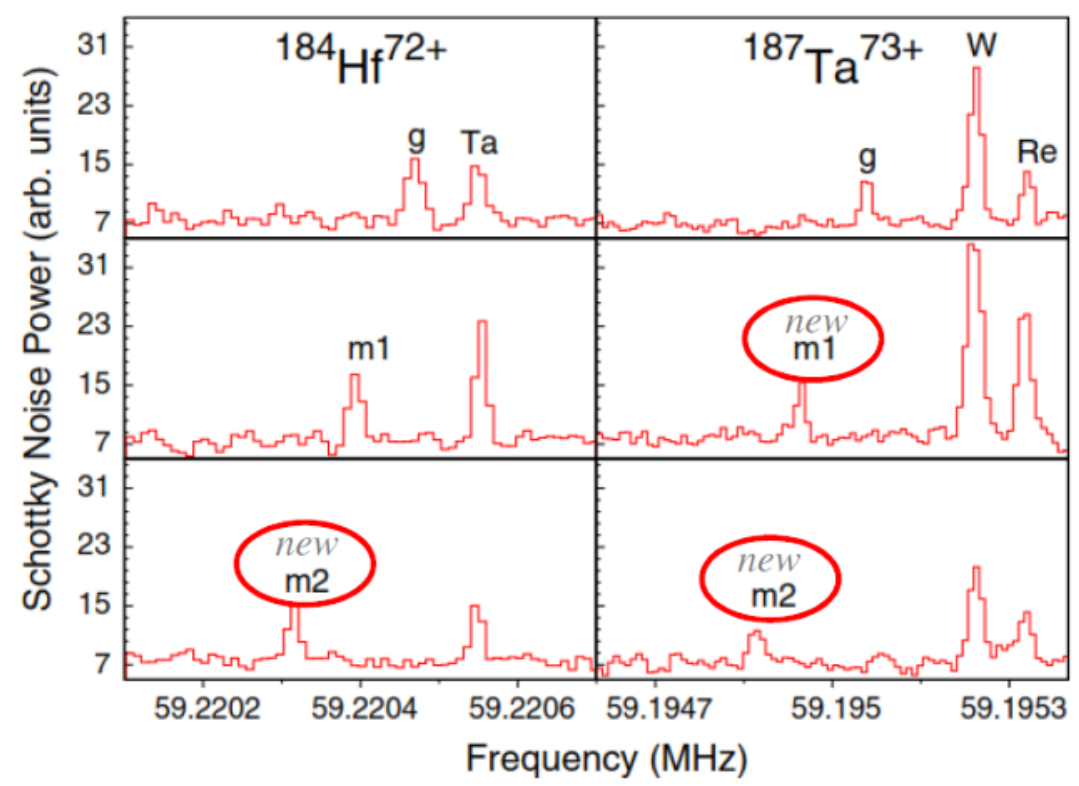

Figure 2. Zoom parts of Schottky frequency spectra exhibit new findings on long-lived isomeric states in midshell isotopes: a new isomeric state in ${ }^{184} \mathrm{Hf}$ (labelled $\mathrm{m} 2$, characterized by a half-life of 12 minutes and an excitation energy of $2477 \mathrm{keV}$ ) and two new isomeric states in ${ }^{187} \mathrm{Ta}$ (for details see [21]).

\subsection{Reactions off internal targets}

A peculiarity of storage and cooler rings are internal targets. Besides special targets (like electron targets) these are mainly ultra-sonic gas-jets, which traverse the ultra-high vacuum and which are used for atomic and nuclear reaction or spectroscopy experiments. The ESR is equipped with gaseous and cryogenic droplet targets of various elements from $\mathrm{H}$ to Xe [22]. Since these targets are characterized by a low areal density (typically of the order of up to $10^{15}$ atoms per $\mathrm{cm}^{2}$ ), they are well suited for studies where the collision kinematics leads to low-energy recoils or ejectiles. This is particularly the case for experiments which require single-collision conditions, for low-energy reactions and/or very peripheral collisions with small momentum transfer. For a given cross section, the low reaction probability, caused by the low target density, can largely be compensated by the multiple revolutions of the ions in a storage ring, thus leading to sufficiently high reaction rates.

The question whether the luminosity reached with external targets (thick targets and single use of the penetrating beam) or with internal targets (thin targets and multiple use of the circulating beam) is superior is rather subtle. Besides the physics to be explored and the setup to be used, the discussion 
essentially boils down to nuclear half-lives and atomic charge-changing processes (mainly due to radiative and non-radiative electron capture from the target). The left part of figure 3 shows a comparison of internal and external reaction rates for various elements and beam energies. Overall, the revolution frequency in the storage ring over-compensates the small number of scattering centers of a thin target, so that for beam energies above $\sim 100 \mathrm{MeV} / \mathrm{u}$ and sufficiently long half-lives the internal target yields higher luminosities than the external target. For low beam energies, heavy elements and short nuclear half-lives the advantages of internal targets become less substantial.

The other key factor is the beam intensity, which can be increased by beam "accumulation" or "stacking". This is a sophisticated sequence of beam manipulations to increase the brilliance of the beam (i.e. increase the intensity and simultaneously reduce the phase space), where the injected exotic nuclei are pre-cooled by stochastic cooling, bunched with rf-cavities, their trajectories are moved from the injection orbit to a storage orbit, where electron cooling then takes over, reduces the longitudinal and transverse emittance and finally merges their orbits with the already stored ions. This process is depicted in a schematic cross-sectional view of the ESR aperture in the upper part of the right hand side of figure 3, while the lower part shows the corresponding Schottky noise signal. The period of such a cycle is approximately 30 seconds. This time can vary from case to case and is a subject for optimization in the future, but it gives an approximate estimate, together with the production yields, of which isotopes (with half-lives of the order of 10 seconds) are in reach for reaction experiments with secondary beams.
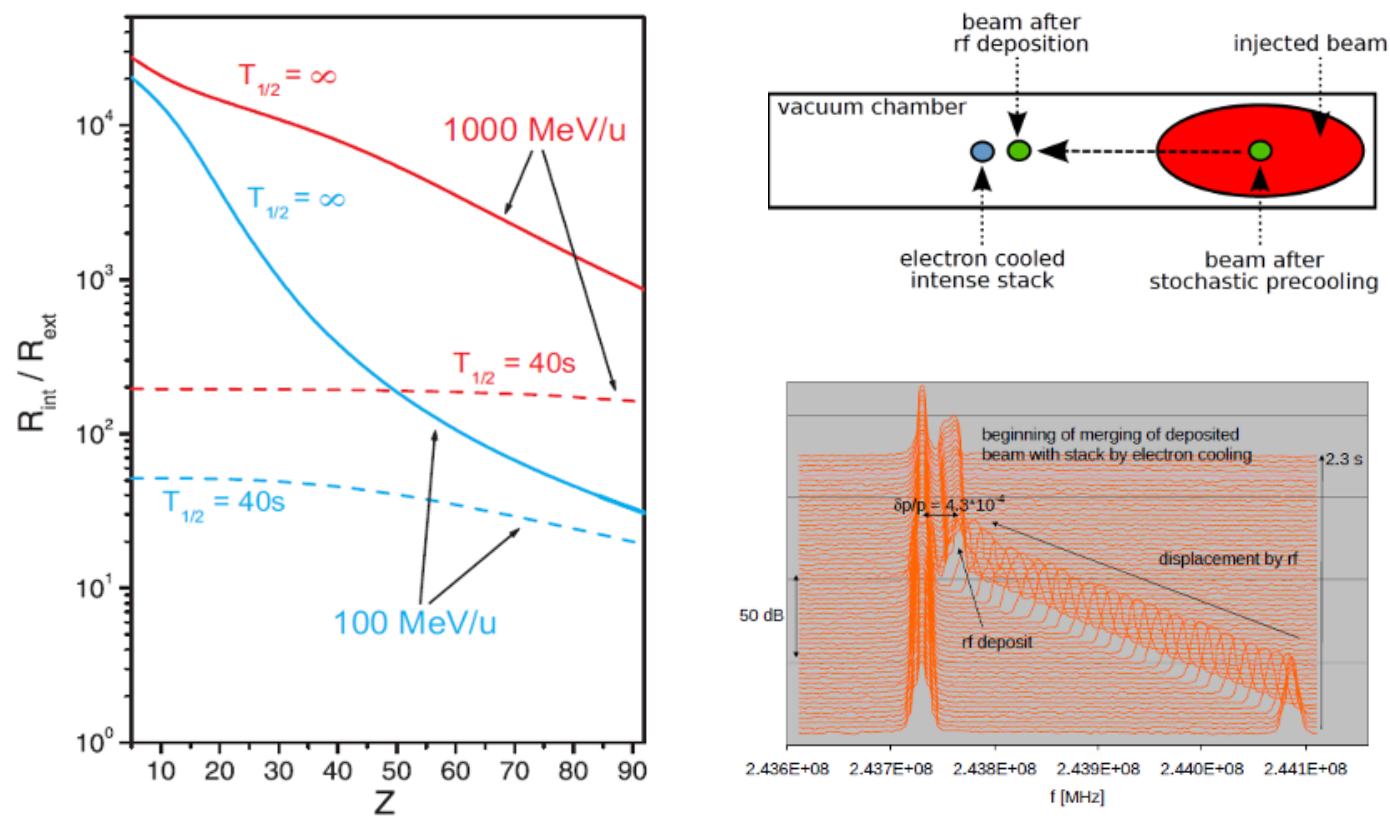

Figure 3. Left: gain factor of internal and external targets versus beam atomic number. The estimation is performed for two different beam energies and for two different half-lives. Obviously internal targets are superior for light ions at high beam energies with long lifetimes. Right: accumulation of ${ }^{56} \mathrm{Ni}$, used for the experiment described below (for details see text). Courtesy: H. Weick, M. Steck, F. Nolden.

For reactions with exotic beams, inverse kinematics is favourable, and the thin internal targets within a storage ring allow one to detect low-energy projectile and/or target recoils, which would suffer from energy and angular straggling in the thick targets used in typical external target experiments. This is the ideal basis for low-momentum transfer collisions. Thus, when aiming at the detection of lowenergy recoils near $90^{\circ}$ in the laboratory frame, which is the case for low-momentum transfer reactions, the thin internal target is clearly unique. Such experiments are e.g. elastic proton or alpha scattering, inelastic proton or alpha scattering, transfer reactions and charge-exchange reactions, 
where the low-momentum transfer reactions contain the most important information. The physics interest is:

- nuclear matter distributions, skin and halo structures,

- deformation parameters, B(E2) values, Coulomb excitation and giant resonances,

- $\quad$ single-particle structure, correlations, capture cross sections and Gamow-Teller strengths.

In a first series of experiments, giant resonances were studied [23]. The various giant resonances probe different properties of the nucleus: the isoscalar giant monopole resonance (ISGMR) probes the nuclear compressibility, the isovector giant dipole resonance (IVGDR) is related to the size of the nucleus etc. In these measurements, the centroid energy and the width of these resonances were obtained. In order to investigate models which describe the underlying dynamics of giant resonances, it is essential to go beyond stable nuclei, and exotic nuclei offer new degrees of freedom (such as isospin, binding energy and different density distributions of protons and neutrons, including a broad range of different neutron densities).

One of the nuclei of key interest is the unstable ${ }^{56} \mathrm{Ni}[24]$. This nucleus is very important for two reasons: first it is a doubly magic nucleus around the $1 \mathrm{f}-2 \mathrm{p}$ shell. Second, it plays a very important role in stellar nucleosynthesis and the formation of the mass-56 peak abundance. An experiment to populate excited states by inelastic proton scattering was proposed by Paul Kienle already in the early $90 \mathrm{~s}$, but it suffered from insufficient luminosity at that time. As shown above, ${ }^{56} \mathrm{Ni}$ beams can be produced and accumulated in sufficient quantity today, so that this new class of reaction experiments with exotic nuclei scattering off internal targets becomes feasible. The first measurements have been performed on ${ }^{56} \mathrm{Ni}$ and the ISGMR and ISGQR have been observed. From the elastic scattering events, matter radii can be obtained with high accuracy $(\sim 0,1 \mathrm{fm})$ and higher moments of the nuclear charge distribution can also be extracted. Preliminary results are described in some detail by M. v. Schmid et al. in a contribution to this proceedings [25].

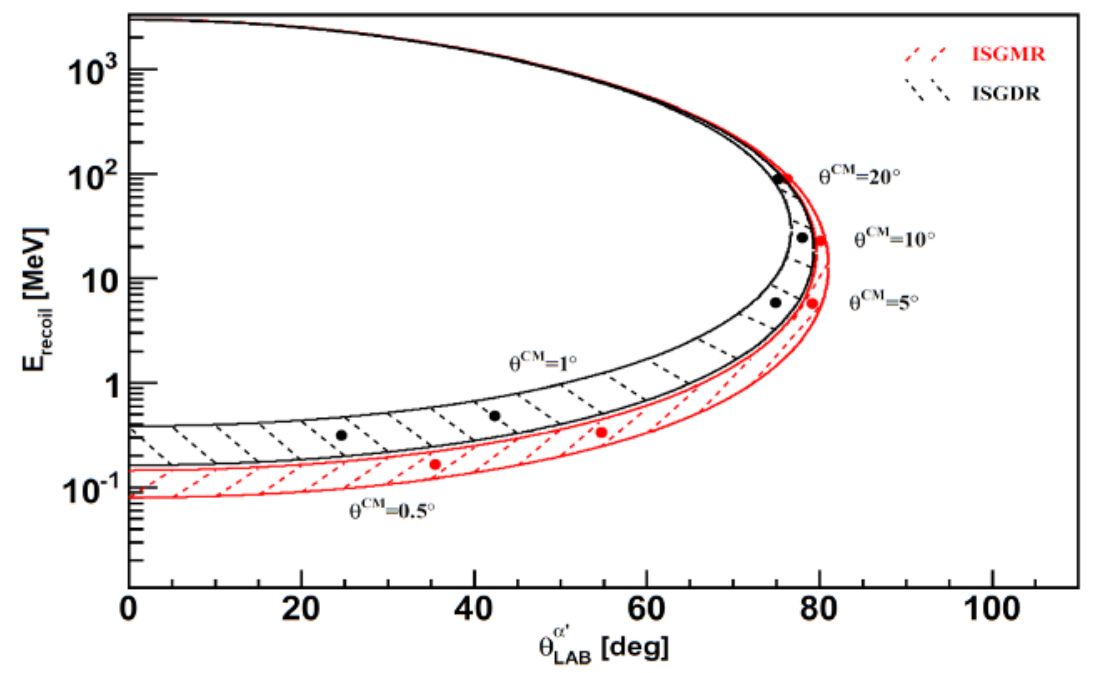

Figure 4. Recoil energy spectrum versus laboratory angle of alpha particles from inelastic scattering in inverse kinematics off ${ }^{56} \mathrm{Ni}$ ions at $200 \mathrm{MeV} / \mathrm{u}$. The red and the black lines show the kinematics for the excitation of the ISGMR and ISGDR. Several c.m. scattering angles are indicated to illustrate the sensitivity for small c.m. scattering angles and small momentum transfers.

At lower beam energies, direct reactions can contribute to explore nuclear input data for stellar and explosive nucleosynthesis. The ultra-thin internal targets allow for single-collision conditions even at small collision energies and also for the detection of low-energy reaction products, and the experiments benefit from the coverage of the full solid angle in the c.m. frame and very low background. 
In a recent experiment the cross section for direct proton capture of stable beam ${ }^{96} \mathrm{Ru}$ was studied [26] at laboratory energies of $10 \mathrm{MeV} / \mathrm{u}$, almost extending to the Gamow-window. While calculations modeling explosive burning scenarios at the outer O-/Ne-layers of a progenitor star during a SN-typeII explosion (where neutron-deficient nuclei are produced by photo-dissociation $(\gamma, \mathrm{n})$ of heavy seed nuclei, followed by $(\gamma, p)$ or $(\gamma, \alpha)$ reactions) can in general reproduce the observed abundances rather well (within a factor 3), the predictions fail for the light p-nuclei such as Mo and Ru by more than one order-of-magnitude. It is therefore important to probe the cross section at a few energies.

Another experiment aimed at the ${ }^{20} \mathrm{Ne}(\mathrm{p}, \mathrm{d}){ }^{19} \mathrm{Ne}$ transfer reaction. The states, which are populated in ${ }^{19} \mathrm{Ne}$, are considered to "regulate" the flow between the hot CNO cycles and the rp-process. In stars, these states are populated in the ${ }^{15} \mathrm{O}(\alpha, \gamma){ }^{19} \mathrm{Ne}$ reaction, which is the nuclear trigger of X-ray bursts and thus a good quantitative understanding is needed for the explanation of amplitude and periodicity of bursts. The key unknown is the $\alpha$-decay probability from the excited state at $4.03 \mathrm{MeV}$ in ${ }^{19} \mathrm{Ne}$ with a predicted probability of the order of $\sim 10^{-4}$ compared to $\gamma$-decay [27].

\section{Conclusions}

The SIS-ESR facility, in combination with the in-flight separator FRS, is the first of its kind to perform experiments with relativistic stored exotic nuclei. Ground-breaking methods and novel detection schemes have been developed, which yielded extremely valuable physics results, which cannot be achieved elsewhere in the world. Along these lines, a series of reaction experiments with internal targets was launched recently. These findings and achievements are the motivation and basis for an even more powerful synchrotron, fragment separator and storage-ring complex at FAIR, which is presently under construction. When the SIS-ESR project at GSI was started in the 80s, such success could not have been forecast. Even more, at that time two essential requirements could not be proven beforehand: that in-flight separation of relativistic beams with shaped matter within ion-optical systems works as well as was predicted, and that electron cooling of highly-charged heavy ions works well without down-charging the ions and losing the beam quickly. In this sense, the present and future success emerges from the dedicated ideas and contributions of many people, some lucky circumstances, and last but not least from the vision, bravery, and determination of Paul Kienle.

\section{Acknowledgements}

The contributions of all members of the various FRS-ESR experiment collaborations over many years are acknowledged. Many thanks for discussions and for providing material to this contribution to F. Bosch, P. Egelhof, H. Geissel, N. Kalantar, T. Kröll, Y. Litvinov, G. Münzenberg, W.R. Plaß, R. Reifarth, P. Walker, P. Woods. We all are full of gratitude to Paul Kienle, who always was a patron of this field, who injected an almost infinite number of ideas and was always an excellent partner for stimulating scientific discussions. We will miss his guidance in the future.

\section{References}

1. J. Dufour et al., Nucl. Instr. Meth. A 248, 267 (1986).

2. G. Münzenberg, H. Geissel, et al., GSI internal report (1986).

3. K. Blasche et al., Joint Accelerator C onference Proceedings of PAC85, 2657 (1985).

4. P. Kienle, Nucl. Instr. Meth. A 271, 277 (1988).

5. H. Geissel et al., Nucl. Instr. Meth B 70, 3412 (1992).

6. B. Franzke, Phys. Scr. 59, 176 (1995).

7. F. Nolden et al., Nucl. Instr. Meth. A532, 79 (2004), and

M. Steck et al., Nucl. Instr. Meth. A532, 357 (2004).

8. H. Geissel et al., Phys. Rev. Lett. 68, 3412 (1992).

9. R. Daudel, Rev. Sci. Instr. 85, 162 (1947). 
10. M. Jung et al., Phys. Rev. Lett. 69, 2164 (1992).

11. H. Irnich et al., Phys. Rev. Lett. 75, 4182 (1995).

12. T. Radon et al., Phys. Rev. Lett. 78, 4701 (1997), and

T. Radon et al., Nucl. Phys. A 677, 75 (2000).

13. M. Hausmann et al., Nucl. Instr. Meth. A 446; 569 (2000), and

J. Stadlmann et al., Phys. Lett. B 586, 27 (2004).

14. B. Sun et al., Phy. Lett. B 688, 294 (2010).

15. H. Wollnik et al., GSI-Report 1, (1985).

16. T. Ohtsubo et al., Phys. Rev. Lett. 95, 052501 (2005).

17. B. Schlitt et al., Hyp. Int. 99, 117 (1996).

18. L. Chen et al., Phys. Rev. Lett. 102, 122503 (2009), and

L. Chen et al., Phys. Lett. B 691, 234 (2010).

19. Yu. A. Litvinov et al., Phys. Lett. B 573, 80 (2003).

20. P. Kienle et al., accepted for publication in Phys. Lett. B.

21. M.W. Reed et al., Phys. Rev. Lett 105, 172501 (2010), and

L. Chen et al., Phys. Rev. Lett. 110, 122502 (2013).

22. H. Reich et al., Nucl. Phys. A 626, 417c (1997), and

M. Kühnel et al., Nucl. Instr.. Meth. A 602, 311 (2009).

23. N. Kalantar-Nayestanaki et al., Int. J. Mod. Phys. E18, 524 (2009).

24. G. Kraus et al., Phys. Rev. Lett. 73, 1773 (1994).

25. M. v. Schmid et al., contribution to this conference.

26. Q. Zhong et al., Journal of Physics: Conference Series 202, 012011 (2010), and R. Reifarth et al., publication in preparation.

27. P.J. Woods et al., publication in preparation. 